Meta

Journal des traducteurs

Translators' Journal

\title{
Eurodicautom : problèmes résolus et problèmes posés
}

\section{Jacques Goetschalckx}

Volume 26, numéro 1, mars 1981

L'informatique au service de la traduction

Machine Aids to Translation

URI : https://id.erudit.org/iderudit/002928ar

DOI : https://doi.org/10.7202/002928ar

Aller au sommaire du numéro

Éditeur(s)

Les Presses de l'Université de Montréal

ISSN

0026-0452 (imprimé)

1492-1421 (numérique)

Découvrir la revue

Citer cet article

Goetschalckx, J. (1981). Eurodicautom : problèmes résolus et problèmes posés. Meta, 26(1), 76-80. https://doi.org/10.7202/002928ar d'utilisation que vous pouvez consulter en ligne.

https://apropos.erudit.org/fr/usagers/politique-dutilisation/ 


\section{Eurodicautom : problèmes résolus et problèmes posés}

JACQUES GOETSCHALCKX

Les institutions européennes occupant plus de 2000 linguistes - traducteurs, interprètes, terminologues - ont compris depuis longtemps l'importance des technologies modernes pour améliorer les outils du traducteur.

La banque de données terminologiques EURODICAUTOM, mise au point par le bureau de terminologie de la Commission à Luxembourg, avec la collaboration de la Direction générale XIII, «Marché de l'Information et Innovation», en ce qui concerne le logiciel, est actuellement gérée par un groupe représentant les deux services précités et le bureau de terminologie de Bruxelles, mais elle est au service de toutes les institutions et organes de la Communauté européenne.

Mise au point par des traducteurs pour des traducteurs - on croit entendre un Lincoln linguiste - c'est un véritable outil électronique au service du traducteur et rien d'autre que cela. Ce n'est pas de la traduction automatique, c'est tout au plus un dictionnaire automatisé.

Fidèles au principe ergonomique qu'il faut adapter l'outil à l'homme et pas l'inverse, les initiateurs d'Eurodicautom se sont efforcés de la structurer en sorte que le traducteur ne soit pas obligé de trop modifier ses méthodes de travail.

C'est ainsi que pour entrer en contact avec le système, l'usager n'a que trois opérations à effectuer :

1) brancher ou faire brancher son terminal

2) entrer dans le système

3) préciser la banque de données qu'il veut consulter, en l'occurrence la banque de terminologie.

Cela se traduit par les trois manipulations suivantes:

allumer le terminal,

faire LOGON, ce qui donne accès à l'ordinateur,

écrire DO TERM, ce qui vous met en liaison avec la banque de terminologie.

À partir de là, le terminal prend l'usager «par la main» pour l'aider à formuler la question qu'il souhaite soumettre à la banque de terminologie. 
La machine lui dit :

spécifiez la langue source et cible(s),

posez votre question.

Si par suite d'une fausse manœuvre, l'usager se trouve en face de réactions inattendues et indésirables du système, une fonction «de secours» lui permet de retomber sur les pattes et de poursuivre son interrogation.

La consultation peut se faire à partir d'une langue source vers une langue cible, ce qui sera la démarche normale du traducteur, ou vers plusieurs langues cibles, ce qui intéresse plutôt le terminologue, le linguiste théoricien et d'autres.

Le dialogue étant établi, quelles sont les informations auxquelles le traducteur peut avoir accès?

L'Eurodicautom offre 4 types d'information:

1) le simple terme à terme, que l'on ne considère que comme un pis-aller mais qui est toujours mieux que rien;

2) des phrases équivalentes, qui offrent des termes difficiles dans une phrase qui peut éclairer l'usager sur les particularités syntaxiques ou sur un certain contexte technique dans lequel ils se placent;

3) des termes assortis de définitions;

4) des termes assortis de contextes illustratifs réels, donc non correspondants d'une langue à l'autre.

Puisque Eurodicautom comprend des entrées phraséologiques, plusieurs mots ne s'y trouvent pas sous leur forme standard. La recherche de ces termes est assurée par le recours à la troncature. Cela signifie que dans cette procédure la machine donne en réponse à la question posée une chaîne de caractères très proche de ceux de la question posée mais différents en ce qui concerne les derniers caractères. C'est un système purement mécanique tant pour les langues germaniques que pour les langues latines et l'anglais.

Une originalité du système est ce que l'on appelle la réponse partielle. Le traducteur qui se trouve confronté avec un syntagme composé de trois ou quatre éléments recherchera d'abord dans le dictionnaire - souvent sans grand espoir - la totalité de l'expression. S'il ne la trouve pas il s'efforcera de retrouver les éléments composants.

Fidèle au principe que l'outil électronique doit suivre ou imiter la démarche normale du traducteur, l'Eurodicautom effectuera automatiquement la même manœuvre. Si vous recherchez le terme «machine à souder, point par point» et que cette expression ne figure pas dans le corpus du système, il vous donnera séparément «machine à souder» et «souder ou soudage point par point».

Pour ceux qui préfêrent le support papier à la vision sur terminal, parce que cela leur est plus familier ou paraît plus tangible et donc plus «solide», l'inter- 
rogation par lots ou l'impression du contenu de l'écran sur la petite imprimante $\mathrm{y}$ associée pourra donner satisfaction.

À ce propos, on pourrait se demander laquelle des deux procédures est la meilleure : interrogation par lots ou en conversationnel. Personnellement, je serais tenté de dire que pour la traduction de gros documents, qui prennent de toute manière plusieurs jours, voire des semaines ou des mois, l'interrogation par lots semble toute indiquée. En revanche, pour la traduction de textes courts et urgents, l'interrogation en conversationnel au terminal s'impose.

Reste à voir quelle est la valeur réelle d'Eurodicautom dans l'état actuel des choses. Il est permis de dire je crois que le système est extrêmement flexible et offre de très larges potentialités.

Mais même si c'était le plus beau système du monde, il se trouverait encore dans la même situation que la femme qui mérite ce même qualificatif.

Ce qui séduit l'usager ce ne sont pas les potentialités ou la flexibilité, mais le taux de réponses obtenu. Or, là il faut de nouveau distinguer les résultats ponctuels et les résultats moyens. Il nous est arrivé d'obtenir un taux de réponses de $95 \%$. Nous étions les premiers à en être étonnés. Malheureusement, les essais effectués sont encore trop dispersés pour avancer un chiffre représentatif pour le taux de réponses moyen, réalisé actuellement.

Fort de l'expérience de certains confrères, nous pensons que la banque de terminologie devrait contenir 400000 à 500000 termes pour obtenir ce qui semble être le taux de réponses moyen qui constitue un seuil difficile à dépasser, à savoir $65 \%$.

L'alimentation d'une banque de terminologie n'est cependant pas simple. En observant le traducteur utilisant une banque de terminologie, on est tenté de dire qu'il cherche :

1) la réponse correspondant exactement à la question posée ;

2) une information absolument sûre.

Ce qu'il n'aime pas c'est :

1) l'apparition de plusieurs réponses possibles:

- lorsqu'elles sont plus ou moins similaires, cela l'irrite;

- lorsqu'elles sont différentes et que les informations complémentaires fournies ne lui permettent pas de trancher avec certitude, il reste perplexe.

2) les pannes d'ordinateur ou de lignes.

Les deux phénomènes se rapportent en fait aux mêmes désirs: régularité et qualité du service.

Les problèmes sous 2 échappent au terminologue. Le problème sous 1 doit cependant retenir toute son attention.

Il s'ensuit que l'introduction de toute nouvelle collection d'informations terminologiques dans la banque nécessite une vérification préalable afin de savoir si les informations à entrer s'y trouvent déjà en tout ou en partie. 
Dans le premier cas, elles sont purement et simplement éliminées afin d'éviter des redondances; dans le second cas il faut fusionner l'ancienne et la nouvelle information en une seule.

C'est là qu'apparaissent les grosses difficultés, surtout lorsqu'il faut travailler en sept langues comme c'est le cas des institutions européennes. Il n'est rien moins que rare que les diverses équivalences offertes par deux ou plusieurs collections terminologiques sont différentes pour une ou plusieurs langues.

Si le terminologue veut s'en tenir au principe "pour chaque concept une seule fiche et une bonne", un idéal pas toujours réalisable, il est obligé de se plonger à son tour dans le sujet et de revérifier chacune des entrées concurrentes. Si elles sont toutes bonnes elles peuvent être traitées comme des synonymes. Les entrées incorrectes seront éliminées. Mais ces recherches sont souvent longues et ne donnent pas toujours un résultat clair et net. Il est évident que si des cas de ce genre sont fréquents on perd une bonne partie de l'avantage qu'il y a à recourir à des collections terminologiques existantes et élaborées par des spécialistes en la matière.

Les travaux de terminologie multilingues mènent très souvent à une remise en question régulière des certitudes acquises précédemment. Pour résoudre les problèmes qui se posent constamment il semble absolument nécessaire d'organiser une collaboration étroite et constante entre linguistes, consommateurs de terrninologie dans des domaines qui leur sont étrangers, et les experts qui ont souvent eux-mêmes beaucoup de peine à suivre les progrès réalisés dans leur domaine, surtout lorsqu'ils travaillent dans une langue autre que celle sous l'empire de laquelle l'innovation a été conçue.

La variété des besoins a donné naissance à divers types de banques de terrninologie. Mais il n'est pas impensable qu'il existe un noyau commun de problèmes terminologiques qu'il faut résoudre pour tout le monde. La différence des systèmes entraîne des solutions distinctes pour ce problème commun également. Là aussi un effort constant de convergence des efforts s'impose donc certainement.

Enfin on ne voit pas pourquoi les banques de terminologie, qui doivent suppléer aux lacunes qui se sont révélées dans les dictionnaires et glossaires, ne pourraient pas établir une collaboration avec les auteurs et éditeurs de ces ouvrages où l'un et l'autre pourrait profiter des informations collectées par son vis-à-vis.

La même démarche pourrait être suivie entre banques de terminologie et systèmes de traduction automatique. Le transfert d'informations posera évidemment des problèmes mais l'ampleur des tâches que nous devons affronter tous devant le vaste problème terminologique ne nous permet pas de laisser inexplorée la moindre veine, quelles que soient les difficultés d'exploitation.

Des efforts énormes sont accomplis pour venir à bout du problème terminologique. On pourrait se demander si c'est justifié. Il me semble qu'il suffit de penser à tout ce qui est encore devant nous. 
À l'heure actuelle, des centaines de millions d'Africains et d'Asiatiques n'ont pratiquement pas encore voix au chapitre parce qu'ils vivent en marge de la société industrielle. Mais cette société dont le berceau se trouve en Europe occidentale, s'est déjà considérablement internationalisée. Non seulement les États-Unis, l'URSS et le Japon mais aussi la Corée et le Brésil et bien d'autres sont devenus des pôles importants.

La conclusion du raisonnement est claire. Nous venons de pénétrer dans cette forêt aux sentiers multiples mais où la végétation devient de plus en plus touffue. 